\title{
Método de integración de stakeholders a procesos de toma de decisiones de las empresas
}

Jorge ROBERTO VOLPENTESTA*

Argentina

\section{Resumen}

Este trabajo presenta un método de integración progresiva y gradual de los stakeholders no accionariales a los procesos decisorios de las empresas, considerando diferentes grados de participación, compromiso e involucramiento.

\section{Introducción}

Los últimos años han mostrado que en la dirección y gestión de empresas cada vez son más las argumentaciones relacionadas con la responsabilidad social empresaria (RSE), la sostenibilidad y los stakeholders, grupos de interés. La incorporación de estos conceptos en la gestión empresarial refiere a ciertos procesos evolutivos en la forma de "ser empresa" que modifica el accionar de aquellas que deciden adoptarlos (Ogliastri et al., 2015).

* Doctor de la Universidad de Buenos Aires, área Ciencias Económicas, subárea Administración. Investigador y profesor titular de la UBA. Contacto: jorge. volpentesta@hotmail.com 
Y dentro de estos procesos, que se muestran multifacéticos y abordables desde diferentes ópticas, existe uno en particular que más impacta en la lógica tradicional de la gestión empresarial: el hecho de que las empresas muestran interés en grupos, organizaciones y personas, las que por mucho tiempo fueron ignoradas por esas mismas empresas. Así, la realidad muestra que en algunas empresas existe la decisión por satisfacer las expectativas, deseos, intereses y demandas de sus stakeholders no accionariales (Langtry, 1994; Donaldson y Preston, 1995; Garicano Rojas, 1998; González Esteban, 2001a; Ogliastri, 2003; Purnell y Freeman, 2012; Alejos, 2015).

El encuadre conceptual de este tema - la teoría de los stakeholders- establece que la incorporación de objetivos de interesados no accionariales con el fin de satisfacerlos, da cuenta de un proceso en el que las empresas que así lo deciden no solo reconocen a sus diversos stakeholders no propietarios y sus intereses, sino que de manera diversa e idiosincrásica habilitan espacios de participación en instancias de su gobierno a través de su intervención en ciertos procesos de toma de decisiones de gestión y de dirección (Rodríguez Fernández, 2006 y 2007).

Los trabajos académicos expresan que la incorporación de objetivos de stakeholders no accionariales a los procesos de gestión, son la representación de la evolución en la forma de ser empresa que algunas de estas compañías han decidido adoptar, de manera que arquetípicamente se las diferencia entre las que tienen un modelo de gobierno accionarial o financiero puro y aquellas con un modelo de gobierno pluralista o gobierno de los stakeholders (González Esteban, 2001a; Stiglitz, 2004; Rodríguez Fernández, 2010).

Para poder dar intervención a los interesados no accionariales en determinados procesos del gobierno empresarial se requiere que entre las empresas y sus stakeholders se habiliten medios de comunicación y espacios de interacción que permitan que las voces de los grupos de interés sean oídas por las empresas y, de alguna manera, resulten atendidos sus intereses y demandas, participando incluso algunos de ellos en determinados procesos de toma de decisiones.

Pero en los mismos ámbitos en los que se manifiesta este fenómeno de gestión - en su praxis empresarial y en los aportes doctrinarios- se puede comprobar que resultan escasos los métodos adecuados 
que indiquen el camino para que esa vinculación se concrete y, de ese modo, se afiance la relación empresa-stakeholders (Ruiz de Aguirre y Retolaza, 2012).

La motivación de realización de este método tiene dos vertientes. Por un lado, la verificación a través de investigaciones de campo (Volpentesta, 2012 y 2014) de que en la práctica empresaria las empresas que quieren generar este tipo de apertura, no saben cómo hacerlo. Por el otro, la constatación, a través de la investigación bibliográfica, de que no existen métodos adecuados para tal fin.

\section{Marco teórico}

Los antecedentes más directos del método propuesto se encuentran en Arnstein (1969) y en Connor (1988), que enfocaron sus trabajos sobre teoría política de empoderamiento ciudadano, y también en Friedman y Miles (2006) así como en el "Manual para la práctica de las relaciones con los grupos de interés de la organización" auspiciado por AccountAbility y escrito por Krick et al. (2006), que refieren exclusivamente a stakeholders de organizaciones, en especial de las empresariales. El modelo propuesto por Friedman y Miles (2006) está basado en el presentado por Arnstein (1969) y, si bien no dice ya que no es un trabajo académico, el de Krick et al. (2006) se basa a su vez en estos dos últimos.

$\mathrm{Al}$ ser los aportes anteriores los antecedentes directos del método propuesto se exponen brevemente sus características más salientes. No sucede lo propio con otros trabajos que también permitieron pensar sobre el método, especialmente los relacionados con redes políticas e interorganizacionales, tales como los de Klijn (1997), Rovere (1999), Zurbriggen (2004) y Martínez Nogueira (2012). Además, se trabajó con material relacionado con el enfoque de la ética dialógica, que sirvieron de inspiración para su elaboración (Cortina, 1990, 2000, 2003, 2005; González Esteban, 2001b; García Marzá, 2003; Argandoña, 2007; Argandoña y Hoivik, 2009).

En su trabajo, Arnstein (1969) argumenta sobre las estructuras de poder en la sociedad y cómo estas interactúan, analizando quién posee 
el poder cuando se toman decisiones importantes. Su modelo de participación ciudadana en el proceso de elaboración de políticas públicas abarca desde los estratos más inferiores que corresponden al paternalismo, hasta los superiores que corresponden al control ciudadano. $\mathrm{Su}$ representación asume la relación entre una comunidad y su gobierno mediante el uso de una escalera como metáfora, la que expresa el aumento al acceso del poder de la comunidad en los procesos de toma de decisiones. Su propuesta busca representar las etapas necesarias para que aquellos ciudadanos excluidos de los procesos políticos y económicos sean deliberadamente incluidos en el futuro.

Por su parte, Connor (1988) presenta un modelo también en escalera, de planificación conjunta entre las personas y los organismos de poder con el propósito de proporcionar un enfoque sistemático para prevenir y resolver controversias públicas sobre políticas específicas, programas y proyectos, ya sean desarrollados solo por los gobiernos como también con participación privada. A diferencia del modelo de Arnstein (1969), este presenta una secuencia temporal para la prevención y solución de conflictos entre partes, en el que cada escalón actúa de manera acumulativa. Aun cuando el modelo no particulariza en los niveles de participación de los ciudadanos, brinda una orientación respecto de la prevención y resolución de conflictos que esa participación podría generar.

En tanto, el modelo de Friedman y Miles (2006) es el primero que se elabora específicamente con la intención de ser aplicado por empresas y para sus grupos de interés. Los autores especifican que es un modelo de compromiso de los interesados que se propone ilustrar los grados cualitativos de la gestión de stakeholders desde la perspectiva de ellos. El modelo distingue doce niveles, y los autores no son partidarios de que todas las relaciones con los stakeholders deban ser llevadas al máximo nivel, o a algún nivel en particular: los distintos grupos de interesados deben ser tratados en diferentes niveles, y luego, un mismo grupo deberá ser tratado en diferentes niveles a lo largo del tiempo. El modelo específicamente es de administración, de manejo de los stakeholders. En su esencia subyace un enfoque estratégico-instrumental, puesto que la idea que prevalece es la de "manejar" 
de alguna manera a los grupos de interés, con un evidente sentido de disminución del riesgo empresario.

Por último, el esquema presentado por Krick et al. (2006) está estructurado en ocho niveles de relación entre las organizaciones y sus stakeholders. Este modelo brinda ejemplos de algunos de los enfoques de relación prácticos más comunes en cada nivel. Cabe recordar que el "Manual para la práctica de las relaciones con los grupos de interés" no es un escrito académico, de manera tal que no identifica las fuentes utilizadas para su realización.

\section{Método de integración de stakeholders}

El método que se propone se fundamenta en escritos y conceptos sobre redes políticas e interorganizacionales y en modelos de empoderamiento ciudadano y gobernanza de empresas, porque la incorporación de stakeholders como participantes activos en determinadas decisiones de las empresas refleja un proceso de empoderamiento que se sustenta en razón de los vínculos que se generan y se van consolidando, precisamente, entre las empresas y sus grupos de interés. Y estos vínculos son entre personas que van elaborando redes personales que se conectan o vinculan, aunque una de esas personas sea el gerente general de una empresa y se relacione con su cargo incluido, porque no se conectan cargos entre sí, no se conectan organizaciones entre sí: se conectan las personas. Y esa construcción de redes tiende a la generación de confianza, materia prima indispensable para sustentar cualquier relación entre las empresas y sus stakeholders.

El método concentra su atención en las actividades y circunstancias que las empresas deberían considerar en su vinculación con los grupos de interés, con el fin de integrarlos a sus procesos de toma de decisiones directivas: enseña acerca de un camino que las empresas pueden seguir para cumplir con dicho objetivo. En ese camino las variables relevantes son la intensidad y la profundidad de la participación de los stakeholders en los procesos de toma de decisiones.

Aun cuando en el entramado final de empresa-stakeholders el nodo principal siguen siendo las empresas representadas por sus integrantes, lo cierto es que la red que se teje explica muchas formas de vinculación 
entre aquellas y sus grupos de interesados y, por ende, el nivel de participación de estos en los procesos empresariales.

A diferencia de otros métodos propuestos por la doctrina, este está fundamentado en el enfoque ético-normativo, porque supone por parte de las empresas la adopción de posturas verdaderas y sinceras de consideración de sus stakeholders como fines en sí mismos y no solo como instrumentos para que otros interesados - por ejemplo, los accionistas- puedan alcanzar sus fines. Y dentro del enfoque ético normativo, tiene su inspiración en los conceptos de la ética dialógica.

Desde la perspectiva del enfoque ético-normativo, el planteo de la ética discursiva o dialógica puede resultar un ideal lejano de alcanzar si se piensa en las diferencias que existen entre los stakeholders y las empresas, las que con sus ajustadas estructuras jerárquicas y su enorme poder informacional y económico, expresan manifiestamente las relaciones asimétricas de poder e igualdad entre ellas y sus stakeholders. La propuesta práctica de instrumentalización de los conceptos de la ética dialógica deberían llevar, entonces, a tratar de nivelar esos desequilibrios de manera que se den las condiciones de representación de todos los stakeholders en un mismo plano de igualdad de oportunidades y simetría. Para que esto se concrete resulta fundamental que los directivos de las empresas asuman que sus grupos de interés son agentes morales, y los incorporen a sus procesos de gobierno a través del diseño de sus estrategias, políticas y decisiones, por medio de mecanismos que aseguren aquellas condiciones.

El método está dirigido a aquellas empresas que realmente creen que la participación de los stakeholders en sus procesos de toma de decisiones directivas y gobernanza representa un valor en sí mismo, una situación que favorece tanto a la empresa como a los stakeholders, considerándolos fines en sí mismos y no simples medios para intentar maximizar los beneficios de una sola clase de interesados. Es por eso que se excluyen del método las ideas que expresan modalidades instrumentales y cínicas de manejo de los grupos de interés. A diferencia de los modelos citados, el método incorpora un nivel de noparticipación en el que las empresas deberían desarrollar determinadas actividades favorecedoras de la posterior y efectiva participación, compromiso e involucramiento de sus stakeholders. Su meta-objetivo es el sincero crecimiento e intensificación 
de los vínculos entre las empresas y sus stakeholders, desde el nivel más superficial hasta lograr su total involucramiento.

Cabe consignar que en la aplicación concreta del método deben tenerse en cuenta las diferentes características de los sistemas de gobierno corporativo que, esquemáticamente, pueden diferenciarse entre los sistemas anglosajón, asiático, germánico y latino (Cuadrado Ballesteros et al., 2015, p. 23). Las características que los diferencian se relacionan con los factores legales, la importancia del mercado de capitales y la estructura de propiedad vigente en cada caso. Vale decir que en función de cada contexto se deberán matizar las recomendaciones propuestas en el método.

\section{Desarrollo del método}

El método formula una progresión en la intensidad del vínculo empresa-stakeholders en cuatro etapas, que se inicia en el momento en el que las empresas toman la decisión de realizar una gestión en la que considerarán los objetivos, intereses, deseos y expectativas de sus stakeholders. A su vez, dentro de cada etapa se distinguen diferentes fases. Luego de una primera etapa en la que las empresas ponen atención en sus grupos de interés, estarían dadas las condiciones para la creación, por iniciativa de ellas, de espacios en donde los stakeholders podrían participar, para luego avanzar a través de un progresivo compromiso de aquellos con determinadas acciones y actividades realizadas conjuntamente con las empresas, hasta desembocar en el involucramiento definitivo de los grupos de interés en determinados procesos decisorios.

El método aspira a ser un referente válido de seguimiento y aplicación para las empresas que quieren empezar a escuchar las voces de sus stakeholders y atender sus demandas. Aunque también puede aplicarse para identificar actuaciones empresarias, encuadrándolas en alguna determinada fase estipulada por el método, así como también reconocer acciones empresarias que no implican un sincero compromiso con sus grupos de interés, las que se ubicarían por debajo del nivel de no participación. 
Cada empresa debería ajustar su camino real de incorporación de sus stakeholders a sus procesos decisorios sobre la base de sus características contextuales y las condiciones espacio-temporales de las vinculaciones ya establecidas, de modo que las etapas y fases que se exponen no necesariamente deben ser desarrolladas en ese orden, así como también alguna o algunas de ellas podrían dejarse de lado. Además, el método tampoco propicia que todos los stakeholders sean llevados a una determinada etapa o fase, pues cada empresa debe determinar en qué etapa o fase desea llevar su vinculación con cada partícipe. A su vez, un mismo grupo de interesados podría estar incluido en diferentes etapas y fases a lo largo del tiempo así como diferentes stakeholders podrían "ingresar" en el método en diferentes fases, debido a que con algunos de ellos las organizaciones tienen habitualmente relaciones, como en el caso de los proveedores y empleados. También pueden existir diferencias si el stakeholder es un actor individual que igualmente puede ser agrupado con otros stakeholders individuales-, se presenta organizacionalmente - sindicatos, ONG- o encarna un colectivo de incierta representación - comunidad.

Las distintas etapas y fases del método de integración gradual de los stakeholders no accionariales a los procesos decisorios de las empresas se muestran en la tabla 1, en el que también se expresan las consecuencias más evidentes del vínculo generado. De manera similar a los modelos tomados como referencia, este método procede de forma escalonada, tal como si cada fase fuese un peldaño que partiendo desde una base determinada llega a la parte superior paso a paso.

En función de la esencia de los vínculos que se generan entre las empresas y sus stakeholders, para su representación gráfica se adopta una imagen que además de mostrar la concatenación de las fases, proporciona la idea de que cada etapa representa una profundización de los vínculos, un continuo entrecruzamiento entre los diferentes actores que participan de la relación. La elección de una figura basada en la grafía del ADN no es casual pues así como este al contener la codificación de las proteínas, y siendo que estas al ser diferentes para cada especie producen que cada una tenga su propia codificación, de manera similar cada empresa tendrá su propia sucesión de pasos para 
llegar a la participación, compromiso e involucramiento de sus stakeholders, y esa sucesión de pasos será diferente a la de otras empresas. La versión gráfica puede verse en la figura 1.

\section{Explicación de sus etapas y fases}

\section{Atención}

A partir de la decisión de tomar en consideración los objetivos, deseos, expectativas e intereses de sus stakeholders, la empresa debería comenzar a realizar determinadas acciones que anticipen y preparen la creación de espacios de participación. Precisamente, la etapa de atención propone la realización de acciones que permitirán que la empresa inicie el camino tendiente a la participación, compromiso e involucramiento de sus stakeholders en ciertos aspectos de su gestión.

\section{Diferenciación}

La diferenciación clara de los stakeholders y sus intereses constituye la base para que la empresa pueda comenzar a transitar con ellos un genuino camino de participación, compromiso e involucramiento, no obstante lo cual esta fase solo meramente expresa un interés instrumental. No hay objetivos explicitados ni más relación que la que podría surgir por las vinculaciones contractuales. No obstante, esta fase es sumamente importante ya que representa, por parte de la empresa, la aceptación de sus stakeholders: si no se acepta que el otro existe difícilmente se genere entre la empresa y sus stakeholders una relación que adquiera profundidad y calidad. Aceptar que el otro existe es asignarle importancia y una entidad propia diferenciada de la de los demás: el otro se hace visible, está presente, de manera que se tiende el primer puente para tomarlo en cuenta. 
Tabla 1. Etapas y fases del método de integración

\begin{tabular}{|c|c|c|c|}
\hline Etapas & Fases & $\begin{array}{l}\text { Consecuencia del } \\
\text { vínculo }\end{array}$ & $\begin{array}{l}\text { Medios e instru- } \\
\text { mentos de vincu- } \\
\text { lación }\end{array}$ \\
\hline \multirow{3}{*}{ Involucramiento } & Control stakeholder & & \\
\hline & Delegación de poder & $\begin{array}{l}\text { Genera y requiere } \\
\text { confianza }\end{array}$ & $\begin{array}{l}\text { Comités de direc- } \\
\text { ción y gestión }\end{array}$ \\
\hline & $\begin{array}{l}\text { Cooperación y aso- } \\
\text { ciación }\end{array}$ & $\begin{array}{l}\text { Genera y requiere } \\
\text { solidaridad }\end{array}$ & $\begin{array}{l}\text { Alianzas estratégi- } \\
\text { cas, sociedades de } \\
\text { riesgo compartido }\end{array}$ \\
\hline \multirow{3}{*}{ Compromiso } & Colaboración & $\begin{array}{l}\text { Genera interde- } \\
\text { pendencia }\end{array}$ & $\begin{array}{l}\text { Proyectos comuni- } \\
\text { tarios, iniciativas } \\
\text { sociales, desarrollo } \\
\text { de proveedores }\end{array}$ \\
\hline & $\begin{array}{l}\text { Conciliación y nego- } \\
\text { ciación }\end{array}$ & $\begin{array}{l}\text { Genera y requiere } \\
\text { compromiso }\end{array}$ & $\begin{array}{l}\text { Técnicas de con- } \\
\text { ciliación y nego- } \\
\text { ciación, técnicas } \\
\text { de análisis de } \\
\text { problemas, cons- } \\
\text { trucción de esce- } \\
\text { narios } \\
\end{array}$ \\
\hline & $\begin{array}{l}\text { Proposición delibe- } \\
\text { rativa }\end{array}$ & $\begin{array}{l}\text { Genera mecanis- } \\
\text { mos de búsqueda } \\
\text { de consenso }\end{array}$ & $\begin{array}{l}\text { Grupos perma- } \\
\text { nentes de asesora- } \\
\text { miento }\end{array}$ \\
\hline \multirow{4}{*}{ Participación } & Diálogo consultivo & $\begin{array}{l}\text { Genera el derecho } \\
\text { de ser informado } \\
\text { y consultado }\end{array}$ & $\begin{array}{l}\text { Rondas de diálogo, } \\
\text { talleres, encues- } \\
\text { tas, reuniones in- } \\
\text { dividuales, focus } \\
\text { group, sondeos de } \\
\text { opinión, grupos de } \\
\text { asesoramiento } \\
\end{array}$ \\
\hline & Diálogo explicativo & $\begin{array}{l}\text { Genera interrela- } \\
\text { ción }\end{array}$ & $\begin{array}{l}\text { Rondas de diálogo, } \\
\text { talleres, encuestas }\end{array}$ \\
\hline & Convocatoria & $\begin{array}{l}\text { Genera la posibi- } \\
\text { lidad de encuen- } \\
\text { tro }\end{array}$ & $\begin{array}{l}\text { Redes sociales } \\
\text { electrónicas, línea } \\
\text { telefónica gratuita, } \\
\text { sitios web, publica- } \\
\text { ciones en medios } \\
\text { de comunicación, } \\
\text { cartelera interna, } \\
\text { bouse organ, in- } \\
\text { tranet } \\
\end{array}$ \\
\hline & $\begin{array}{l}\text { Reconocimiento mu- } \\
\text { tuo }\end{array}$ & $\begin{array}{l}\text { Genera y requiere } \\
\text { reciprocidad }\end{array}$ & $\begin{array}{l}\text { Visitas a la em- } \\
\text { presa, visitas a los } \\
\text { stakeholders }\end{array}$ \\
\hline
\end{tabular}


Sección I. Responsabilidad Social Empresarial (RSE)

\begin{tabular}{|c|c|c|c|}
\hline Etapas & Fases & $\begin{array}{l}\text { Consecuencia del } \\
\text { vínculo }\end{array}$ & $\begin{array}{l}\text { Medios e instru- } \\
\text { mentos de vincu- } \\
\text { lación }\end{array}$ \\
\hline \multirow{5}{*}{ Atención } & Asistencia & $\begin{array}{l}\text { Genera un primer } \\
\text { espacio de articu- } \\
\text { lación conjunta } \\
\text { empresa-stake- } \\
\text { holder }\end{array}$ & $\begin{array}{l}\text { Contribuciones re- } \\
\text { queridas }\end{array}$ \\
\hline & Seguimiento & $\begin{array}{l}\text { Genera voluntad } \\
\text { de construcción } \\
\text { conjunta }\end{array}$ & $\begin{array}{l}\text { Revisión activa y } \\
\text { constante de: car- } \\
\text { tas, expresiones en } \\
\text { medios de comuni- } \\
\text { cación e Internet, } \\
\text { protestas, buzón } \\
\text { de sugerencias, in- } \\
\text { vestigaciones de } \\
\text { mercado }\end{array}$ \\
\hline & Información & $\begin{array}{l}\text { Genera transpa- } \\
\text { rencia y legitimi- } \\
\text { dad }\end{array}$ & $\begin{array}{l}\text { Conferencias, pre- } \\
\text { sentaciones públi- } \\
\text { cas, publicaciones, } \\
\text { publicidad en me- } \\
\text { dios de comuni- } \\
\text { cación, reportes } \\
\text { de sustentabilidad } \\
\text { "sinceros" }\end{array}$ \\
\hline & Conocimiento & $\begin{array}{l}\text { Genera interés en } \\
\text { los otros }\end{array}$ & $\begin{array}{l}\text { Cartas, expresio- } \\
\text { nes en medios de } \\
\text { comunicación e In- } \\
\text { ternet, protestas }\end{array}$ \\
\hline & Diferenciación & $\begin{array}{l}\text { Genera acepta- } \\
\text { ción de los otros }\end{array}$ & $\begin{array}{l}\text { Mapeo de stake- } \\
\text { holders }\end{array}$ \\
\hline
\end{tabular}

Fuente: elaboración propia. 
Figura 1. Etapas y fases del método de integración

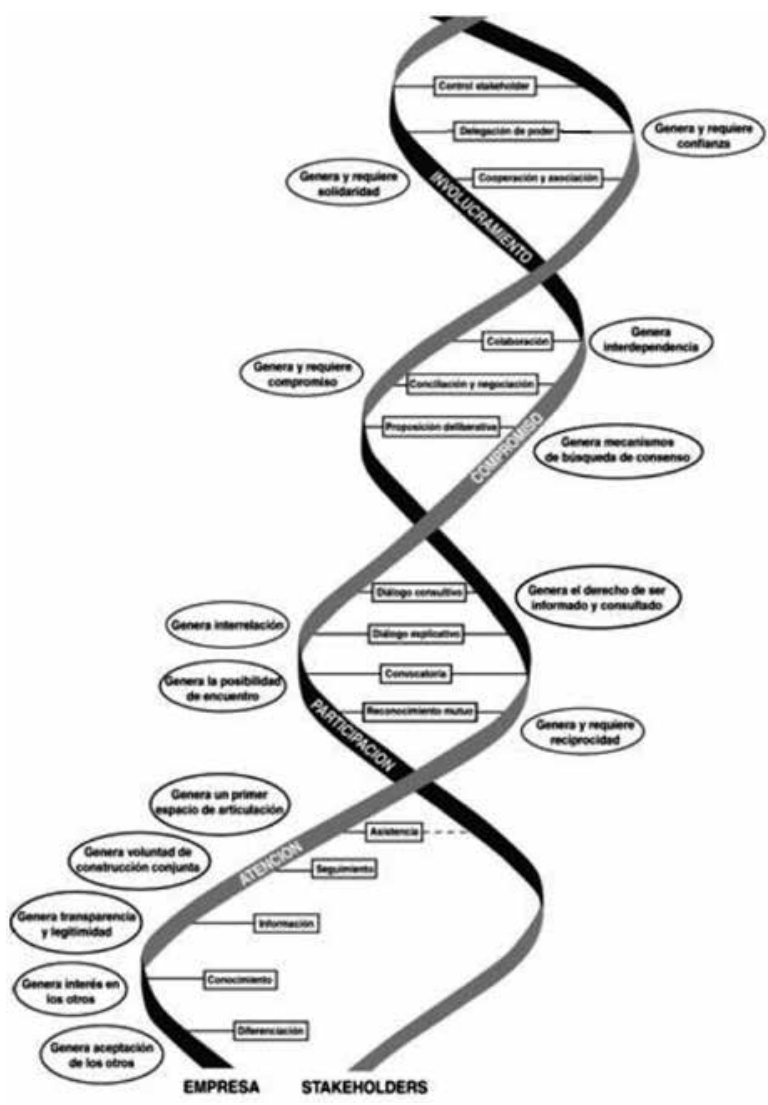

Fuente: elaboración propia.

\section{Conocimiento}

Las empresas podrían diferenciar a sus stakeholders pero no necesariamente tener el conocimiento de ellos. Esta fase supone la manifestación de la inclinación del ánimo por parte de la empresa hacia cada uno de sus stakeholders: se expresa, de esta manera, que cada uno de ellos “importa” por sí mismo para la empresa. Y representa un esfuerzo por parte de la empresa para tratar de entender la posición del otro, el lugar desde donde el otro observa la realidad y expresa sus opiniones. 


\section{Información}

Las empresas podrían liberar información a los stakeholders de manera abierta y transparente. Esta información sería unidireccional ya que no espera respuesta por parte de los stakeholders-puesto que tampoco se los invita a responder-, y podría ser dirigida como no dirigida intencionalmente a un stakeholder en particular.

\section{Seguimiento}

El seguimiento consiste en ir tras de lo que opinen los stakeholders, estableciendo así el primer eslabón en el que la empresa manifiesta que tiene interés en un conocimiento activo de sus stakeholders, y que no solo le interesa tener la "foto" de ellos en un determinado momento - propio de la fase de conocimiento - sino que su intención consiste en dotar a la relación del dinamismo propio que posee toda vinculación.

\section{Asistencia}

La asistencia supone un espacio de encuentro, si bien esporádico, entre la empresa y sus stakeholders; a la vez representa la primera instancia en la cual la comunicación fluye de uno a otro, de manera bidireccional. Las acciones que se concretan en esta fase resultan fundamentales para que los stakeholders sientan que pueden confiar en la empresa a la vez que esta realiza acciones que tienden a consolidar el vínculo. Se genera un primer espacio de articulación conjunta en una situación concreta entre la empresa y sus stakeholders, aun cuando la asistencia puede circunscribirse a uno solo de ellos y desaparecer luego de la acción.

\section{Participación}

Participar es "estar", “intervenir”, en un tiempo y lugar determinados; estar e intervenir no necesariamente implica que quien participa tenga obligación alguna, o la necesidad de realizar ciertas acciones por haber participado. Participar de algo o tomar parte en alguna cosa o asunto es reconocer la no posesión de esa cosa o algo: no se participa de aquello que uno mismo posee. De manera que, ineludiblemente, la participación es cosa de dos, y representa algo más que la consulta respecto 
de medios y fines: es un proceso en el cual dos partes o más se influyen mutuamente en la realización de planes, programas y objetivos.

\section{Reconocimiento mutuo}

Si bien en la fase de conocimiento se discriminaba a los stakeholders por la naturaleza, cualidades y relaciones de las cosas, en esta fase de reconocimiento el examen cuidadoso y detenido se produce entre ambas partes. Expresa el conocimiento en profundidad de algo o de alguien; en lenguaje platónico, significa sacar a la luz el verdadero conocimiento de algo.

\section{Convocatoria}

Una vez que existe el reconocimiento mutuo, cualquier integrante del espacio en el que se produce la participación está en condiciones de efectuar una convocatoria para realizar diferentes actividades. Aun cuando el método asume que la empresa es el sujeto activo que impulsa la participación de los stakeholders, una vez que estos tienen definido el espacio de participación, también están en condiciones de generar esta solicitud.

\section{Diálogo explicativo}

Aun cuando en esta fase ya se encontrarían habilitados los canales bidireccionales de comunicación y se tendría consciencia de la existencia de espacios de participación, el diálogo explicativo permitiría a la empresa informarle a sus stakeholders, previo a su anuncio público, sobre decisiones que ya hubiesen sido tomadas.

\section{Diálogo consultivo}

Sobre una agenda de temas dispuestos por la empresa se convoca a los stakeholders para conocer sus puntos de vista. La empresa busca obtener información y feedback de los stakeholders como insumo de sus procesos de toma de decisiones. Para ello se los escucha con atención y se toman en cuenta sus apreciaciones en las decisiones que se estuvieren considerando. 


\section{Compromiso}

Existe dentro del compromiso un componente sustancialmente importante que es el de la existencia de una relación. El compromiso significa: compartir con otros sin perder la identidad; tener visión de futuro en conexión con la realidad; trabajar para transformar la realidad, optimizándola. El compromiso así conceptualizado expresa un estadio superior al de la participación, ya que supone la realización de acciones conjuntas entre aquellos que han aunado sus voluntades y se prometen seguir un camino que lleve al logro de los objetivos establecidos de común acuerdo, coordinando sus acciones a través del ajuste mutuo de sus voluntades.

\section{Proposición deliberativa}

Esta fase del método representa el momento en el cual a los stakeholders no solo se les informa y consulta sino que también se les invita a aportar ideas y plantear propuestas sobre los temas que sean de su interés. Y si es la empresa la que asume la iniciativa de proponer, debe saber que esta instancia deja abierta la posibilidad de que a las propuestas se les introduzcan modificaciones que los stakeholders formulen.

\section{Conciliación y negociación}

La consulta verdadera efectuada a los stakeholders a través del diálogo consultivo y la proposición deliberativa puede llevar a que los puntos de vista entre estos y la empresa sean divergentes, e incluso, contrapuestos. Antes de llegar a una decisión final, a la empresa le va a interesar eliminar, o bien disminuir tanto como sea posible, los factores discordantes o contradictorios. Para ello la empresa deberá conciliar y negociar con sus stakeholders.

\section{Colaboración}

La colaboración refiere a un trabajo en conjunto con otra u otras personas para realizar una obra entendiéndose que representa una ayuda que se presta para que otro/s pueda/n lograr algo que, de otra manera, no hubiera/n podido hacer o le/s hubiera costado más. A diferencia de la asistencia, en esta fase el compromiso se va asumiendo 
progresivamente por ambas partes de modo que nada que lo que una haga le resulta indiferente a la otra.

\section{Involucramiento}

En la etapa de compromiso los stakeholders aceptan los objetivos y la visión de la empresa, pero en esta etapa de involucramiento esos objetivos son compartidos por los stakeholders en un grado mayor de compenetración, de empatía con la organización; en parte esto surge porque en esta etapa la empresa fija sus objetivos volcando en ellos muchos de los objetivos, deseos, expectativas e intereses de sus stakeholders. Para que exista involucramiento los stakeholders deben entender con claridad el propósito de las acciones que van a emprender, habiendo previamente identificado y consensuado con la empresa los intereses, deseos y expectativas que esperan ser satisfechos a través de acciones claras y definidas.

\section{Cooperación y asociación}

Entre la fase de colaboración y ésta de cooperación y asociación existe una profundización mayor en el vínculo empresa-stakeholders. El factor diferencial que permite discriminar con mayor precisión estas fases es que en esta, el trabajo conjunto se verifica desde el inicio del proyecto, desde la determinación misma de los objetivos: genera acciones y decisiones conjuntas. Aquí el proyecto no tiene un único "dueño" sino que desde su misma génesis ambas partes intervienen a través de un importante involucramiento mutuo, en la medida que se verifique la existencia de una finalidad explícita de interés para las partes, que a su vez no reemplace ni colisione con los fines propios de quienes participan, ni con las funciones o estructuras formales existentes.

\section{Delegación de poder}

Esta fase del método de integración de los stakeholders en los procesos decisorios de las empresas implica la existencia de una representación significativa de aquéllos en los órganos decisorios de la empresa, tanto de gestión como de dirección. En este punto hay que diferenciar la participación de los stakeholders en órganos consultivos o de 
asesoramiento por invitación de la empresa, de aquellas situaciones en las que los stakeholders participan efectivamente en cuerpos colegiados que tienen la responsabilidad de la toma de decisiones en algunas temáticas de la gestión empresarial.

\section{Control stakeholder}

Esta fase representa una situación excepcional en la cual los stakeholders pasarían a detentar la mayoría - o todas- las posiciones que toman decisiones así como el control total del proceso, lo que equivaldría a poseer el completo control administrativo en una empresa así como el acceso y control sobre los recursos. En una sociedad capitalista la noción de control absoluto por parte de los stakeholders resulta una situación verdaderamente excepcional. 


\section{Referencias}

Alejos, L. C. (Octubre, 2015). Gobierno Corporativo ¿y? responsable. Cuadernos de la Cátedra "la Caixa" de Responsabilidad Social de la Empresa y Gobierno Corporativo, N. ${ }^{\circ} 28$.

Argandoña, A. (2007). Responsabilidad social de la empresa: ¿qué modelo económico? ¿qué modelo de empresa? Documentación social, 146, 11-24.

Argandoña, A. y Hoivik, H. (2009). Corporate social responsibility: one size does not fit all. Collecting evidence fron Europe. Journal of Business Ethics, 89(3), 221-234.

Arnstein, S. R. (julio, 1969). A ladder of citizen participation. Journal of the American Institute of Planners, 35(4), 216-224.

Connor, D. M. (1988). A new ladder of citizen participation. National Civic Review, 77(3), 249-257.

Cortina, A. (1990). Ética sin moral. Madrid: Tecnos.

Cortina, A. (1994/2000). Ética de la empresa. Madrid: Editorial Trotta S.A.

Cortina, A. (2003). Construir confianza: ética de la empresa en la sociedad de la información y las comunicaciones. Madrid: Editorial Trotta SA.

Cortina, A. (20 de agosto de 2005). Ética de la empresa, no solo responsabilidad social. El País, edición impresa. Recuperado de http://elpais.com/ diario/2005/08/20/opinion/1124488806_850215.html

Cuadrado Ballesteros, B., García Rubio, R., y Martínez Ferrero, J. (2015). Efecto de la composición del consejo de administración en las prácticas de responsabilidad social corporativa. Revista de contabilidad, Spanish Accounting Review, 18(1), 20-31.

Donaldson, T. y Preston, L. E. (1995). The stakeholder theory of the corporation: Concepts, evidence, and implications. The Academy of Management Review, 20(1), 65-91.

Friedman, A., y Miles, S. (2006). Stakeholders: theory and practice. Oxford: Oxford University Press.

García-Marzá, F. (2003). Confianza y poder: la responsabilidad moral de las empresas de la comunicación. En A. Cortina. Construir confianza (pp. 195-220). Madrid: Editorial Trotta S.A.

Garicano Rojas, T. (Marzo, 1998). Los distintos modelos y actitudes ante el gobierno de la empresa: stockholders y stakeholders. Información Comercial Española, 769, 23-34. 
González Esteban, E. (2001a). Consecuencialismo y deontologismo para una ética empresarial: una mirada desde la teoría de stakeholders. Sociedade em Debate, 7(1), 31-45.

González Esteban, E. (2001b). La responsabilidad moral de la empresa: una revisión de la teoría de stakeholder desde la ética discursiva. (Tesis de Doctorado). Facultad de Ciencias Humanas y Sociales, Departamento de Filosofía y Sociología, Universitat Jaume I de Castellón. Castellón.

Klijn, E. H. (1997). Policy networks: an overview. En Kickert, W. J. M., Klijn, E. H. y Koppenjan, J. F. M. (Eds.), Managing complex networks. London: Sage Publications Ltd.Recuperado de http://revista-redes.rediris.es/ webredes/textos/Complex.pdf

Krick, T., Forstater, M., Monaghan, P. y Sillanpää, M. (2006). El compromiso con los stakeholders. Manual de práctica de las relaciones con los grupos de interés. Accountability, United Nations Environment Programme, Stakeholder Research Associates Canada Inc. Recuperado de http://www. accountability.org/images/content/2/0/204.pdf

Langstry, B. (1994). Stakeholders and the moral responsibilities of business. Business Ethics Quarterly, 4(4), 431-443.

Martínez Nogueira, R. (2012). Las redes públicas y las redes interorganizacionales: los problemas de coherencia y coordinación. Voces en el Fenix, Año3, N. ${ }^{\circ} 18,48-53$.

Ogliastri, E. (2003). Alianzas cívicas y la empresa social: una introducción. Revista Latinoamericana de Administración, 31, segundo semestre de 2003, 5-13.

Ogliastri, E., Prado, A., Jäger, U., Vives, A. \& Reficco, E. (2015). Social Business. In James D. Wright (editor-om-chief), Internacional Encyclopedia of the Social \& Behavorial Sciences, 2nd edition, Vol 22. Oford: Elsevier, 68-173.

Purnell, L. S. y Freeman, R. E. (2012). Stakeholder theory, fact/value dichotomy, and the normative core: how wall street stops the ethics conversation. Journal of Business Ethics, 109(1), 109-116.

Rodríguez Fernández, J. M. (diciembre de 2006). La responsabilidad social de la empresa: ¿un medio o un fin? Revista de Dirección y Administración de Empresas, N. ${ }^{\circ} 13,53-77$.

Rodríguez Fernández, J. M. (2007). Responsabilidad social corporativa y análisis económico: práctica frente a teoría. Ekonomiaz, N. ${ }^{\circ} 65,2^{\circ}$ cuatrimestre, 12-49. 
Rodríguez, J. M. (mayo-agosto, 2010). ¿Cómo formulamos la misión y visión de la empresa para que sea compatible con al RSE y el buen gobierno? Revista de la Responsabilidad Social de la Empresa, N. ${ }^{\circ}$, 1-4, [Versión digital].Recuperado de http://www.fundacionluisvives.org/rse/digital/5/ articulos/53794/index.html

Rovere, M. R. (1999). Redes en salud: un nuevo paradigma para el abordaje de las organizaciones y la comunidad. (Reimpresión). Rosario: Ed. Secretaría de Salud Pública.

Ruiz De Aguirre, L. S. y Retolaza, J. L. (2012). Participación de los stakeholders en la gobernanza corporativa: fundamentación ontológica y propuesta metodológica. Universitas Psychologica, 11(2), 619-628.

Stiglitz, J. (2004). Business social responsibility. En BSR 2004 Annual Conference, New York.Recuperado de http://www.bsr.org/BSRConferences/2004/ keynotes/2004_jstiglitz.pdf.

Volpentesta, J. R. (2014). Identificación del impacto de la gestión de los stakeholders en las estructuras de las empresas que desarrollan estrategias de responsabilidad social empresarial. Revista Universidad \&Empresa, 26, 63-92.

Volpentesta, J. R. (julio-diciembre, 2012). Las acciones sociales en empresas con responsabilidad social. Revista Visión de Futuro, 16(2), 105-125, Año 9.

Zurbriggen, C. (octubre de 2004). Redes, actores e instituciones. Reforma y Democracia, N. ${ }^{\circ} 30$. Recuperado de http://www.clad.org/ portal/publicaciones-del-clad/revista-clad-reforma-democracia/ articulos/030-octubre-2004/0051000 